\title{
BUSINESS PROCESS MODELLING FOR ACADEMIC VIRTUAL ORGANIZATIONS
}

\author{
Paul Cotofrei ${ }^{1}$, Kilian Stoffel ${ }^{2}$ \\ ${ }^{I}$ Information Management Institute, University of Neuchâtel, paul.cotofrei@unine.ch \\ ${ }^{2}$ Information Management Institute, University of Neuchâtel, kilian.stoffel@unine.ch
}

SWITZERLAND

\begin{abstract}
The increasing mobility due to Bologna process forces the academic partners to increase the inter-operability of their administrative processes, by interacting through a collaborative networks and therefore acting as an academic virtual organization. To facilitate the communication and the comprehension of the administrative processes between the components of the CN, the Business Process Modeling Notation (BPMN) is proposed in this paper as a standard graphical model for administrative processes and transactions. The adaptability of this standard for academic processes and the difficulties of "translating" the actual administrative models (legal texts) in $B P M N$ diagrams are analyzed.
\end{abstract}

\section{INTRODUCTION}

One of the goals of the Bologna process [1] - an intergovernmental initiative which aims to create the European higher education area by making academic degree standards and quality assurance standards more comparable and compatible throughout Europe - is the increasing of student's and teacher's mobility (inside a country and across countries). Consequently, the institutions of the educational area (universities, engineering colleges, high schools of applied science,...) must respond by an increasing inter-operability of their administrative processes, acting as a single higher educational virtual organization. Those institutions (academic partners) often interact through collaborative networks.

These virtual organizations must offer services to its "customers": course subscription, exam registration, library application, etc. (for students), curriculum recognition, session exam results management, etc. (for the administrative staff), educational program implementation, pre-requisite course preparation, etc. (for the teaching staff). And each partner of the academic virtual organization (AVO) often still has the tendency to protect its autonomy by implementing its own workflows for these services. The realization of collaborative administrative processes and of their support systems in this "multi-enterprises" context becomes so an important issue. Consequently, academic partners must "re-think" their administrative process organisation to find the suitable implementation of these services. A first important step in this direction can be attainted by modelling and decomposing recursively these processes, which are turned into appropriate services either by adding a convenient interface directly, or by associating them to composed services. 
Even if today the universities are forced to operate in a globalising market with powerful new competitors (e.g. open universities), and are pushed by financial necessities to become "hybrid" institutions (semi-public and semi-private), their management is in our regions still not identical to an enterprise management (at least at the strategic level) [2]. Usually, the model of an administrative process (the process which, into a classical enterprise context, would be identified as an "operational business process"), is in written form, mostly plain text, with semantic constraints - laws, faculty regulations, department regulations, education office directives, dean directives, secretary rules, etc.(see Section 2). This form is far-away to be easily comprehensible, even for in-house students or administrative staff, and do not facilitate the collaboration inside AVO.

Different studies were conducted concerning the management of business processes in collaborative networks, which propose a number of frameworks for process modelling to facilitate the partnerships across units that have been traditionally autonomic to large extent [3-4]. But in many cases, these studies concentrated on the technological aspects (WSDL [5], WSDL-S [6] for describing services; SOAP [7], REST [8] for communicating) of the interoperability among heterogeneous tools and platforms [9-10].

Based on the studies of collaborative processes for virtual enterprises [11-12] and on the intuitive equivalences: "university $\approx$ enterprise" and "administrative process $\approx$ business process", we investigate in this paper the suitability of the Business Process Modelling Notation to represent administrative processes inside an AVO. BPMN is a standardized graphical notation that depicts the steps in a business process and facilitates the understanding of high-performance collaborations and administrative transactions between the organizations. By applying a "translating" procedure (in this incipient phase, demanding a strong implication of the user, but intended to become, in the future, an almost automatic procedure), we obtained BPMN diagrams for some of most useful (and used) services (see Section 3). These diagrams were then used in a survey study, designed to establish the degree of comprehension (measured as the rate of correct answers) of the textual model (the regulation), respectively of the corresponding graphical model (see results in Section 4). The encouraging results of our study proved the feasibility of the proposed approach and permitted the opening of the second phase of our project, the creation of a knowledge base of BPMN diagrams for all services supplied by academic partners in a AVO, by using a formal, ontology based, "translation" procedure of the textual models (see Section 5 for a detailed discussion).

\section{MOTIVATING EXAMPLE}

The University of Neuchâtel participates in different exchange programs, either at a national level (BeNeFri and "Triangle d'azur" programs with the universities of Lausanne, Geneva, Fribourg and Bern) or at an international level (Erasmus Program, with universities inside the EU, and specific programs, with universities from the USA and Canada). We propose as illustration the following situation: suppose that a student enrolled in a mobility program wants to attend a course of the 
program "Master of Science in Information Systems" of the Faculty of Economics, University of Neuchâtel.

The academic procedures he must follow in order to obtain his ECTS credits are specified in different legal texts - regulations, rules and instructions - that the student has to understand in order to follow the correct administrative procedure. In any academic unit the models of the administrative processes are represented in written forms with semantic constraints (the terms used in these texts have precise meaning and can not be interpreted outside a specific ontology). Usually we can differentiate three levels of the model specification: a "strategic" level (the process is formulated in a general manner, or even only named), a "tactic" level (the process is described more precise and the agents (rolls) are enumerated), and an operational level (containing the detailed description of the tasks, decisions, agents implied in the process and eventually the connection with other processes).

We will now illustrate the steps a student has to follow and, at the same time, will present the texts "describing" these steps. The first procedure the student must execute is the registration for the chosen course. The legal text containing the specification of the process model at strategic level is the "Regulation of Studies for the Master of Science in Information Systems" [13]:

"Students must register for the examination session..." (art. 9, par. 3).

There is no mention about the registration for a course, the dependence between the two kinds of registration procedures being made clear only at the tactic level of the process model, in the "Rules for attendance and examination of the Bachelor's degree in economic sciences" [14]:

"Students must register for each course they want to attend, within the time prescribed by the academic calendar..." (art. 10, par. 1)

"Registration for a course gives the right to register to the corresponding assessment." (art. 10, par. 2)

How the student may register in practice is described at the operational level of the process model, in the document "Instructions concerning registration for courses and examinations" [15]:

"...It is not allowed to register for an exam without having pre-register for the course ... Joint registration courses/exams is not technically possible, you must register twice.... Please go to page http://www2.unine.ch//academia and log you in with your user name and password. You can find instructions for general use..." (art. 2, par. 1)

"Remember to save your registration before leaving the page. Print your registration to keep a written record" (art. 2, par. 4)

According to the detailed instructions, the registration for a course is performed using a web service (IS-Academia), which demands firstly an authentication phase. A student enrolled at e.g. the University of Lausanne for the Master in Information Systems (which is a collaborative program between the universities of Neuchâtel and Lausanne) has no user name and password for IS-Academia. He must firstly find and then follow the supplementary instructions for this specific case [16]:

1 We use these terms in order to make allusion to the corresponding businesses process levels. 
"Complete the form Enrolment Application - Courses at UniNE and return it to the Secretariat of the Faculty of Economics, with a registration certificate of your university. You will receive a letter giving you a username and password in order to access the university's email system.."

This is only an example of the difficulties a student participating in an exchange program may face trying to achieve all the administrative procedures demanded by the host institution. Furthermore, the details of some academic processes remain obscured even for the teaching or the administrative staff. This is true especially for those processes with a large number of tasks and roles: the creation of a master program, for example, implies the institute which propose the master, the expert group which elaborate the proposal, the council of the faculty which vote the proposal, the dean's office which elaborate the final proposal and the university council which analyse and approve the program.

\section{IMPLEMENTING ACADEMIC PROCESSES}

Academic processes modeled as legal texts do not facilitate the collaboration between the academic partners of a virtual organization. A better approach, in our opinion, is the use of a standard graphical notation for academic processes, readily understandable by all the implied actors: students, professors, secretaries, etc. The standard we chose was designed for business process and is called Business Process Modeling Notation [17]. BPMN provide businesses with the capability of understanding their internal business procedures in a graphical notation and give organizations the ability to communicate these procedures in a standard manner. Furthermore, the graphical notation facilitates the understanding of highperformance collaborations and business transactions between the organizations.

The modeling in BPMN is made by simple diagrams with a small set of graphical elements. The four basic categories of elements are the following:

Table 1 - Elements of BPMN

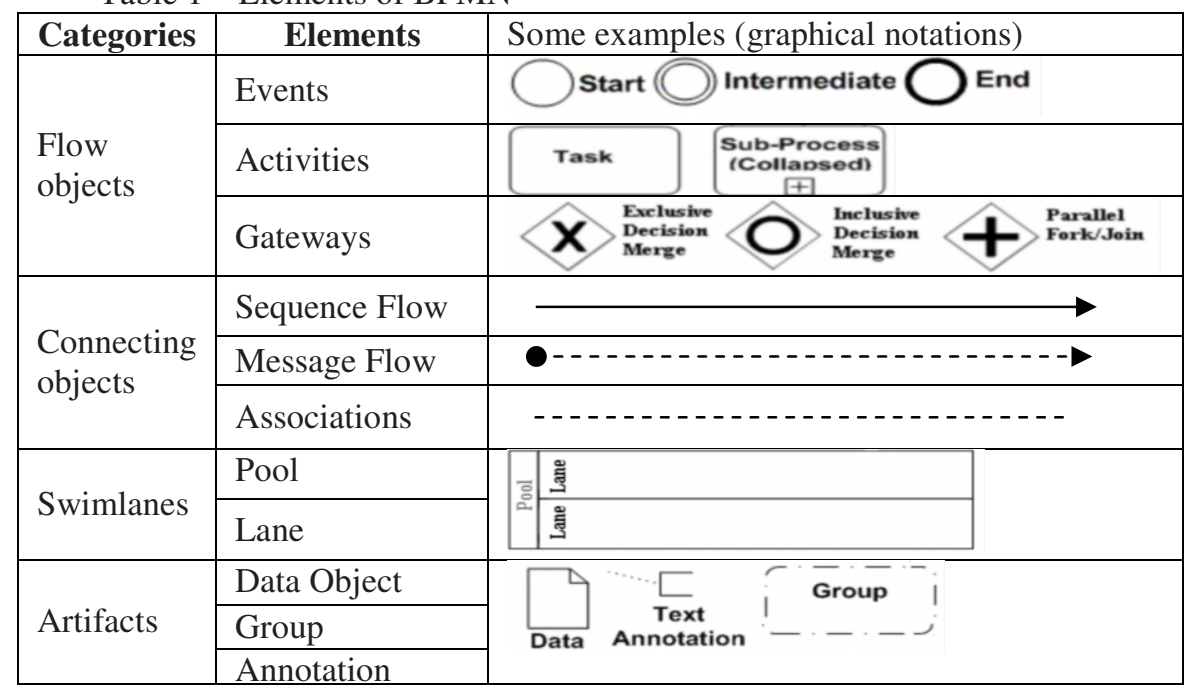


By using these elements, we were able to represent the academic process "Course registration", as is shown in Figure 1.

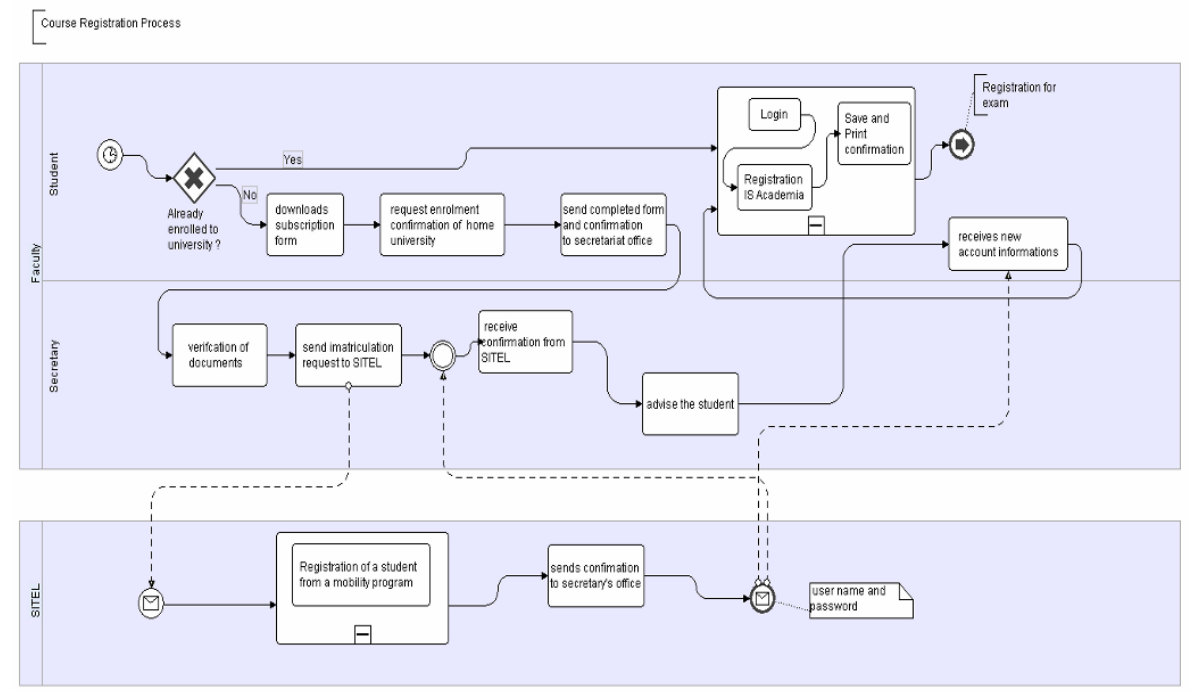

Figure 1. The BPMN diagram of the academic process "Course registration"

Almost all the graphical elements enumerated in Table 1 have been used in this diagram: pools ${ }^{2}$ ("Faculty", with two lanes (or roles) - "student" and "secretary" and "SITEL"), Start Events (of type "Timer" (see Figure 2) - the process is trigged by a specific date from the academic calendar - and of type "Message" - the process is trigged by a received message), End Events (of type "Link" - the process' end will trigger another future process - and of type "Message" - a message is send to a participant), Tasks (e.g. "downloads subscription form"), Sub-processes (e.g. "registration of a student from a mobility program"), Gateway (of type "Exclusive decision/merge", e.g. "Already enrolled to university?"), Sequence Flows, Message Flows, Data and Annotations.

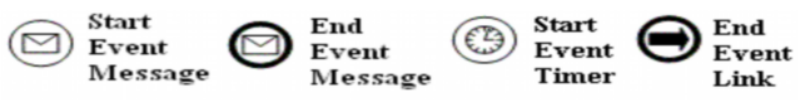

Figure 2. Graphical notation for some specific events

As mentioned in its specifications, BPMN is constrained to support only the concepts of modeling that are applicable to business processes. Academic institutions are organizations with non-business purposes, but the type of services they offers are still equivalent to the services implemented by any common business-oriented organization for its customers. Consequently, the expressiveness of BPMN is sufficient to allow the modeling of any of the academic processes.

2 A pool represents a participant in the process 
From a methodologically point of view, modeling an academic process using BPMN starts by a deeper analyse and comprehension of the process workflow. This task is realized by a person knowing well the semantics of BPMN diagrams, either by analyzing the relevant legal texts or by interrogating administrative staff (especially when the process is known only as a usual practice). The authors' experience showed that some complex administrative processes (e.g. implementtation of a new educational program) are not completely "covered" by regulation texts - the decisional approach for some particular situations being learned only by practice and being lost if the executing person is replaced (especially if this person belongs to the academic staff).

The diagram in Figure 1 was realized using the graphical editor tool included in SOA Tools Platform (STP) project from the open development platform Eclipse [18]. This choice was influenced by the existence of a build-in BPMN verification tool, which checks permanently the concordance of the model with BPMN specifications.

\section{SURVEY RESULTS}

To evaluate the degree of comprehensibility of BPMN diagrams for the common users of academic processes, we conducted a small survey study. Firstly, a randomly sample of 57 students $^{3}$ (including also persons participating in mobility programs) was divided randomly in two groups. One of the groups received the text of the regulations' articles concerning the registration process; the other group received the BPMN diagrams of the same process (the diagram represented in Figure 1 and another, not represented here, for the exam registration process). All the students received also an identical questionnaire, with questions about specific details of the registration process and questions concerning the general appreciation - a note between 1 and 5 - of the process model (text or diagram). At the end, all students received both models and were asked to indicate their preference. A similar study, but less structured and at a very small scale, was conducted for the administrative staff (the secretaries of the Faculty of Economics and the members of the dean's office of the same faculty). In this last study the questions concerned the academic process of the creation, implementation and maintenance of an educational program.

The analysis of the questionnaires (Table 2) showed a rate of preference for BPMN diagrams twice greater than those of regulation texts, even if there are not great differences between the rates of correct answers (or general appreciation) for the two models. Furthermore, the diagrams submitted to administrative staff allowed to bring out some aspects of the academic process, not enough detailed in legal texts.

Table 2. Some results of the survey concerning the BPMN diagrams

\begin{tabular}{|l|c|c|}
\hline & BPMN diagrams & Regulation texts \\
\hline Rate of accurate answers & $87 \%$ & $71 \%$ \\
\hline Positive appreciation (per group) & 3.4 & 3.7 \\
\hline Global preference (per sample) & $48 \%$ & $22 \%$ \\
\hline
\end{tabular}

${ }^{3}$ Enrolled in the first year of a master programs, only $30 \%$ graduated in Neuchâtel 


\section{DISCUSSIONS AND FUTURE WORK}

The advantages of BPMN as a standard modeling language for administrative processes in an academic virtual organization are obvious: easily understandable by users without business background (students, administrative staff, teaching staff), high expressiveness (sufficiently to model any kind of administrative process), userfriendly graphical tools for diagram creation. Furthermore, the possibility of mapping a BPMN diagram into a BPEL processes facilitates the implementation of collaborative processes between academic partners based on Web Service interfaces.

The procedure applied to extract a graphical model (BPMN diagram) from unstructured legal texts originating from different repositories, demands certains abilities from the user (knowledge about the administrative structure of an academic institution and of the relationships between the members of the academic hierarchy, juridical capabilities, and BPMN specifications). So this procedure is only conceivable when applied to construct a small number of models.

For a large number of administrative models (hundreds for the University of Neuchâtel) we suggest another approach (which is briefly outlined here and is part of our planed future work). In a first phase, an ontology of the legal texts defining the structure and the activities of an academic institution is created (usually, these texts employ terms with non-ambiguous meaning). Two distinct categories must be emphasized in this ontology: the hierarchy of the actors (roles) inside the institution, and the activities (tasks) each actor has the competence to execute. In a second phase, a user-driven procedure identifies possible processes by exploring the relationships defined in the ontology (applying either a top-down approach - starting with very general processes and checking if the executed tasks can be expanded into sub-processes - or a bottom-up approach - starting with atomic processes, collapsing them and checking if more general processes can be identified) and transforms them to an "intermediate" model. We believe that the concept of social networks [19-20] could represent a satisfactory solution for this intermediate model. It can be represented in a graphical form (as a finite state machine), captures the behavior of the process (the roles and their actions) and can model the same process at different levels of details, using building blocks [21]. Finally, during the third and last step, an automatic procedure transforms the social networks in BPMN diagrams, using a dedicated knowledge base of equivalences (an example is presented in Figure 3, where at the left we have a social network model and at right the corresponding BPMN diagram).

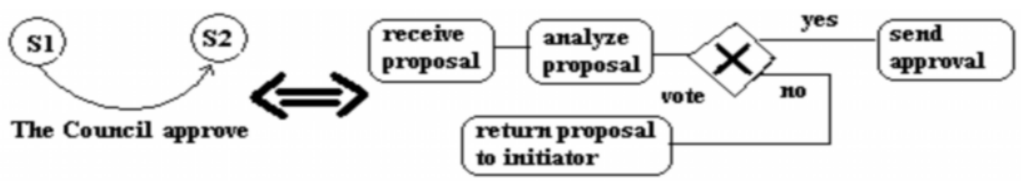

Figure 3. Two equivalent models (at left, a social network and at right, a BPMN diagram, for the administrative process "Approval by the Council") 


\section{CONCLUSIONS}

The collaboration process inside a virtual organization, between non-business institutions for which the models of administrative processes are represented by legal texts, may be facilitate, in our opinion, by adopting BPMN as standard process modeling notation. Our analyze proved the adaptability of this approach for academic processes and proposed to study, while stressing the inherent difficulties, a user-driven, ontology based procedure for "translating" textual models into BPMN diagrams.

\section{REFERENCES}

1. Confederation of EU Rectors' Conferences. "The Bologna Declaration on the European space for higher education". http://ec.europa.eu/education/policies/educ/bologna/bologna.pdf

2. Shattock, Michael. "Strategic Management in European Universities in an Age of Increasing Institutional Self Reliance". In Tertiary Educational and Management, vol.2, no. 6, 2000, 93-104

3. Krogstie, John; Dalberg, Vibeke; Jensen, Siri Moe. "Harmonising Business Processes of Collaborative Networked Organisations Using Process Modelling". In Proceedings of PRO-VE 2004, Toulouse, 81-88

4. Biennier, Frédérique; Favrel, Joël. "Integration of a Contract Framework in BP Models". In Proceedings of PRO-VE 2004, Toulouse 2004, 97-104

5. WSDL link. http://www.w3.org/TR/wsdl

6. WSDL-S http://www.w3.org/Submission/WSDL-S/

7. SOAP link http://www.w3.org/TR/soap/

8. Fielding, R. T. "Architectural Styles and the Design of Networked-based Software Architectures". Doctoral dissertation, Chapter 5, University of California, 2000

9. Carreras, M. A.; Skarmeta, M.; Gomez, A. F. "Towards Interoperability in Collaborative Environments". In Proceeding of "Collaborative Computing: Networking, Applications and Worksharing", 2006, 1-5

10. Franco, Rubén Darío; Bas, Ángel Ortiz; Anaya, Víctor; Esteban, Francisco-Cruz Lario. "IDR: A Proposal for Managing Inter-Organizational Business Processes by Using Web-Services Oriented Architectures". In Proceedings of PRO-VE 2004, Toulouse 2004, 89-96

11. Westphal, Ingo; Thoben, Klaus-Dieter; Seifert, Marcus. "Measuring Collaboration Performance In Virtual Organizations". In Proceedings of PRO-VE 2007, Guimaraes, 33-42

12. Robinson, P.; Karabulut, Y.; Haller, J. "Dynamic virtual organization management for service oriented enterprise applications". In Proceedings of International Conference on Collaborative Computing, 2005.

13. "Master of Science in Information Systems - Règlement d'études". http://www2.unine.ch/webdav/site/mscis/shared/documents/reglement-final-MScIS.pdf

14. "Règlement d'études et d'examens du baccalauréat universitaire en sciences économiques". http://rsn.ne.ch/ajour/default.html?4163301.htm

15. "Inscriptions aux cours et aux examens : Bachelor en sciences économiques 2007-2008". http://www2.unine.ch/webdav/site/seco/shared/documents/PROCEDURE_Exa_etudiants0708.pdf

16. "For students registered at UNIL and UNIGE (Triangle Azur) and following courses at the University of Neuchâtel". http://www2.unine.ch/seco/page21606.html

17. "Business Process Modeling Notation Specification". http://www.bpmn.org

18. Eclipse link http://www.eclipse.org

19. Picard, Willy. "Computer Support for Adaptive Human Collaboration with Negotiable Social Protocols". In Proceeding of BIS 2006, 90-101

20. Picard, Willy. "Continuous Management Of Professional Virtual Community Inheritance Based On The Adaptation Of Social Protocols". In Proceeding of PRO-VE 2007, 381-388

21. Simon, E.; Künzi, C; Stoffel, K. "Scalable Social Protocols to Formalize Systems Development Life Cycles", In Proceedings of IADIS International Conference e-Society 2007, 177-184. 